\title{
ACCESS TO TELESCOPES IN DEVELOPING COUNTRIES
}

\author{
J.B.HEARNSHAW \\ Mt John University Observatory \\ Dept. of Physics and Astronomy, University of Canterbury \\ Private Bag 4800, Christchurch, New Zealand \\ e-mail: j.hearnshaw@phys.canterbury.ac.nz
}

\section{Introduction}

The ambiguity in this paper's title is deliberate. Are we talking about astronomers or telescopes in developing countries? And are the countries concerned developing economically or astronomically? In practice I will cover both options in each case.

The list below summarizes six possible routes for the acquisition of astronomical observational data that may be available to astronomers in countries developing economically, astronomically or in both ways. Not all these options will be available in any one case; indeed most of them will probably not be available. However, examples of all of them can readily be found.

1. Travel to a major observatory to collect data, possibly in N. America

or Europe, Chile or Australia.

2. Acquire an old telescope from a developed country and relocate it.

3. Receive a donation from a developed country for the purchase of a small telescope, or the gift of a small telescope.

4. Obtain data from an overseas country over the Internet

a) other people's data downloaded from a database of observations

b) data obtained as the result of service observing, possibly from an automated

telescope, or a network of such telescopes.

5. Negotiate with a developed nation to establish an observatory in the developing country to which local astronomers have some access.

6. Build a telescope using the resources available in the developing country and establish an observatory.

\section{Pros and Cons of the Different Solutions}

Option 1 is relatively inexpensive in this era of mass travel. Most observatories give free visitor access to telescopes but may charge for accommodation. It is easily the most practised option, though the funding for such trips constitutes a continuing overhead cost of employing an astronomer. In some countries funding for one-off capital costs may be obtained more readily than annually recurring overheads for travel. Expertise in instrumental development is not fostered. Moreover, the data, once obtained, must be analyzed. Normally that requires a computing facility at the home institution.

Option 2, that of transferring obsolete technology to a host nation, has fortunately been rarely practised, and is strongly not recommended. If a developed country finds an instrument to be past its useful working life for research, it is likely that a developing country will find it even harder to obtain useful results from such an instrument. One example of such a donation was the 25-inch Newall refractor (built 1871) in England, which went to the National Observatory in Athens in 1956. In New Zealand we acquired the 18-inch Brashear refractor (1897) from the Flower Observatory in 1963 , but thankfully funds to erect an expensive building for it were never realized.

Donations of small telescopes are occasionally made to developing countries, for example a 45-cm Goto reflector from the Japanese government to Sri Lanka in 1995. Both Indonesia and 
Vietnam have earlier received similar telescopes from Japan in this programme. Such instruments are usually small, but ideal for establishing a university teaching programme in astronomy, though with good instrumentation and reduction software, a useful observing programme may be possible. Many developing countries are located in tropical latitudes and lack good observing sites. But for a small teaching instrument this may not be considered a major obstacle.

The Internet is the least expensive option for acquiring data. For the reduction of large CCD images, an adequate computing facility must be available, which may often not be the case in developing countries. This option gives no hands-on expertise in instrumentation and telescopes, so one of the major benefits in training astronomers is completely absent. Nevertheless data transfers via FTP on the Internet to developing countries are likely to become increasingly important in the future. Most major observatories archive their observations in a database which may be available to others. In addition automatic photometric telescopes can now undertake service observing for astronomers at remote and distant sites and send the reduced data for interpretation to those making the observing request. There is a proposal for a Global Network of Automated Telescopes (GNAT: see http://www.gnat.org/) undertaking CCD photometry using about six telescopes of aperture 0.5 to $1.0 \mathrm{~m}$. If established, this could become an important research tool for astronomers in developing countries.

Very few developing countries today possess such outstanding astronomical sites that they can attract major observatories from overseas to their soil. Chile is the outstanding modern example, but over the last century Peru, South Africa, Bolivia and Indonesia also provided notable examples. In New Zealand, Mt John was founded in 1965 as a southern station of the University of Pennsylvania, though today it is entirely operated by the University of Canterbury. Future new projects of this type are likely to be confined to only the very best observing sites and will be an option for very few developing nations.

Constructing one's own telescope entirely from local resources, expertise and funding may be an option for a few economically developed but astronomically developing countries. This was the situation in New Zealand when we embarked on our 1-m telescope project in 1980. There are possibly several countries in the world with the necessary expertise, infrastructure and funding where this becomes a viable option, which can cost substantially less than the purchase of a completed instrument obtained commercially. Possibly several ASEAN countries, and smaller non-ESO countries in Europe may be in the position to build telescopes in the $0.5-\mathrm{m}$ to $1.5-\mathrm{m}$ size range. The major benefits of so doing are the saving of costs and that it places great emphasis on developing instrumental expertise in mechanical and optical fabrication, in electronics and computer control, all of which are skills which have implications beyond astronomy.

Many astronomers have in recent years expressed concern about the disappearance of small telescopes of about 1-m aperture in developed countries, as facilities are closed down in order to divert financial resources towards major new telescope projects. For this reason the acquisition and operation of small telescopes in developing countries could become a major contribution to variable-star research in future years. Much astronomy of this type is the ideal domain for small telescopes, especially if they are in a developing country where the scheduling and operations are controlled locally.

It is possibly true that any observational astronomy in developing countries is best undertaken in a university environment rather than at a separate government-funded observatory. The training of students is a vital activity in any developing country, and there are many benefits of having a continuous flow of new students becoming acquainted with astronomical observing, a very few of whom may later become professional astronomers, rather than a small and permanent staffing complement devoted solely to research. This has certainly been our experience in New Zealand.

\section{The Mt John 1-m telescope project.}

The 1-metre telescope project was initiated entirely within the Department of Physics and Astronony at Canterbury. The Dall-Kirkham optics were undertaken in collaboration with the Physics and Engineering Laboratory at NZ's DSIR. Field correcting lenses gave the $\mathrm{f} / 8$ focus a 1.7 -degree field. This is the largest wide-field Dall-Kirkaham telescope in the world, and the optical configuration is the only unconventional aspect in the design. It was chosen because of ease of optical fabrication and testing (the secondary being speherical), and the demonstration that image quality 
is almost as good as that of a Ritchey-Chretien. The mirror blanks were Schott Zerodur. Nearly all the mecahnical construction, plus all the electronics and computer control were undertaken within the department. Apart from the optics, it is essentially a conventional single-pier equatorial telescope with $\mathrm{f} / 8$ or $\mathrm{f} / 13.5$ Cassegrain foci. Also part of the project was construction of the 8 -m dome (wood plus aluminium skin) and mirror aluminizing plant. The building and rising floor were handled by the university, not the department. The only major items purchased from overseas were the mirror blanks from Germany and two sets of precision worm and wheel gears from Pennsylvania.

Construction was commenced in May 1981, and the project was essentially complete at the end of 1985. The telescope was installed in February 1986. The work took about 12 man-years of technician time.

The total funding was $\$ N Z 223,400$, raised between 1979 and 1984 . We had estimated $\$ N Z 200,000$ (1981 dollars; in that year \$US1.00 was about equal to \$NZ1.00) so this was essentially within budget. This figure includes the cost of parts and materials for telescope, dome and aluminizing plant, but not for labour or overheads. Funding came from several recurring grants from the NZ University Grants Committee (now defunct), NZ Lottery, University of Canterbury, the Department of Physics and Astronomy and from a public appeal. The last raised $10 \%$ of the cost from 95 private and commercial donors.

The success of the telescope can be ascribed to having all the necessary expertise available locally, good departmental workshops and sufficient (but still limited) funding. The cost of a completed instrument from overseas would have been about a million dollars. The telescope has been in continuous operation for the last eleven years with very little down-time. It has been used for high resolution echelle spectroscopy (Cassegrain and fibre fed), for medium resolution spectroscopy and for CCD and photoelectric photometry. Much of the observatory's work is on variable stars.

If the telescope had been designed only a few years later, we would probably have selected a radically different mounting and drive system. An alt-az mount, a friction drive in both coordinates and a thinner primary (our Zerodur blank was $136 \mathrm{~mm}$ thick at the edge and weighed $275 \mathrm{~kg}$ before central-core trepanning) would all have saved costs and possibly improved performance as well. However, the Dall-Kirkham optics have been very successful, and are an option worth adopting at other small telescopes. 